\title{
Validación del autoinforme de motivos para la práctica del ejercicio físico con adolescentes (AMPEF): diferencias por género, edad y ciclo escolar \\ Validation of the self-report of reasons for the practice of physical exercise with teenagers (AMPEF): differences by gender, age and school year \\ José Domínguez Alonso, Antonio López Castedo, Iago Portela Pino \\ Universidad de Vigo (España)
}

\begin{abstract}
Resumen. El objetivo del estudio es la validación del Autoinforme de Motivos para la Práctica del Ejercicio Físico (AMPEF) de Capdevila, Niñerola y Pintanel (2004), y comprobar los efectos que producen el género, edad y ciclo escolar sobre dichos motivos en una muestra de 852 adolescentes gallegos $(M=14.9 ; D T=1.75)$. Se realizó un análisis factorial exploratorio y confirmatorio obteniendo una adaptación de la escala original de 48 ítems agrupados en ocho factores con excelentes coeficientes de fiabilidad $(\alpha=.96)$, una varianza total explicada del $65.24 \%$ y un ajuste adecuado a los datos $\left(\mathrm{x}^{2} / \mathrm{df}=2.635, \mathrm{GFI}=.909, \mathrm{CFI}=.901, \mathrm{RMSEA}=.047\right)$. Asimismo, los adolescentes que presentan una mejor motivación del factor urgencias de salud (único significativo en las tres variables) son de género masculino, de temprana edad (12-13 años) y primer ciclo de la ESO. También, se aprecia una mejor motivación de los hombres en los factores competición, diversión y fuerza. Por último, se discuten los resultados, destacando el autoinforme para la práctica del ejercicio físico (AMPEF) como un instrumento válido y fiable para valorar el tipo de motivación desarrollado por los adolescentes hacia el ejercicio físico.
\end{abstract}

Palabras clave. Autoinforme, ejercicio físico, motivación, adolescencia, validación, fiabilidad.

Abstract. The objective of the study is to validate the Self-Report of Motives for the Practice of Physical Exercise (AMPEF) by Capdevila, Niñerola and Pintanel (2004), and to verify the effects of gender, age and school cycle on these motifs in a sample of 852 Galician teenagers $(M=14.9$; $D T=$ 1.75). An exploratory and confirmatory factor analysis was performed, obtaining an adaptation of the original scale of 48 items grouped into eight factors with excellent reliability coefficients $(\alpha=.96)$, a total explained variance of $65.24 \%$ and an adequate adjustment to the data $\left(\mathrm{x}^{2} / \mathrm{df}=2.635\right.$, GFI $=.909$, CFI $=.901$, RMSEA $=.047$ ), adolescents who present a better motivation of the health emergency factor (it is the only significant in the three variables) are male, early age (12-13 years) and first cycle of Compulsory Secondary Education. Also, a better motivation of men than woman in the factors of competition, fun and strength can be appreciated. Finally, the results are discussed, emphasizing the self-report for the practice of physical exercise (AMPEF) as a valid and reliable instrument to assess the type of motivation developed by adolescents towards physical exercise.

Keywords. Self-report, physical exercise, motivation, adolescence, validation, reliability.

\section{Introducción}

La incidencia y prevalencia de la obesidad y el sobrepeso están aumentando en todo el mundo. De ahí que la planificación de la promoción de la salud deba tener en cuenta no solo los conocimientos de los adolescentes sobre la importancia de la actividad física sino también las percepciones, motivaciones y necesidades de esta población(Wilkenfeld, Pagnini, Booth, \& King, 2008; Morente, Zagalaz, Molero, \& Carrillo, 2012).

De hecho, la evidencia científica señala que el movimiento corporal es un factor esencial de la actividad física para contrarrestar el sedentarismo. Un meta-análisis realizado por Wilmot et al. (2012), relacionó comportamientos sedentarios (largos periodos de tiempo sentados) con el doble de probabilidad de padecer diabetes o problemas del corazón. Igualmente, el estar demasiado tiempo sentado en el trabajo, viendo la televisión, el uso del ordenador, videojuegos, entre otros, socava el bienestar emocional (Atkin, Adams, Bull, \& Biddle, 2012), puede producir trastornos psicológicos (Kilpatrick, Sanderson, Blizzard, Teale, \& Venn, 2013) y depresivos (Van Uffelen et al., 2013).

Los estudiantes con un nivel más alto de actividad física suelen ser aquellos que tienen un alto nivel de motivación intrínseca y el alumnado que participa activamente en las clases de educación física suelen ser más activos por lo que estas clases representan una oportunidad para que los educadores puedan revertir las tasas de inactividad física entre los jóvenes (Trites, \& Elgar, 2010). De hecho, se está promoviendo la inclusión de la actividad física en los recreos escolares ya que incluso este tiempo de actividad puede tener importantes implicaciones para la salud y para el desarrollo físico, social y cognitivo (Serra, 2014). La Organización Mundial de la Salud (OMS, 2010) recomienda: realizar actividad física durante sesenta minutos al día, como mínimo, con intensidad moderada o vigorosa; ejecutar actividades aeróbicas; $y$, al menos tres veces por semana, actividades que refuercen los músculos y los huesos.

Fecha recepción: 28-06-17. Fecha de aceptación: 05-10-17 José Domínguez Alonso jdalonso@uvigo.es
Así pues, entre los factores que pueden elevar la motivación hacia la práctica del ejercicio físico se han señalado como determinantes, la presión de grupo, algunos videojuegos (Kumar et al., 2016), acceso fácil a un gimnasio, dirección inicial por un entrenador experto, variedad de actividades o deportes en equipo, participación de los apoyos familiares y entre compañeros (Peeters et al., 2012), o la sensación de satisfacción después de la pérdida de peso (Vergara, Santibáñez, Herrera, \& Argote, 2012). En consonancia, los factores motivadores más importantes parecen ser el grupo de amigos, el entrenador y la escuela (Kimberly, Hultquist, \& McLester, 2013; Perry, \& Hoffman, 2010). Para Nicholson et al. (2013) en un estudio con pacientes que han sufrido un accidente cerebrovascular los motivadores más comúnmente reportados son el apoyo social y la necesidad de poder realizar tareas diarias. Por su parte, Mailey, Huberty, Dinkel, y McAuley (2014), en un estudio con padres, señalan que los principales facilitadores de la actividad física son el estar activo con los niños o durante las actividades de los hijos, convertirse en un modelo para los hijos, buscar tiempo y priorizar los beneficios para la salud y la familia y disponer de apoyo. En concreto, su estudio con padres manifiesta que sus prioridades han cambiado y ahora dedican más tiempo a la familia. Entre las actividades más motivadoras figuran los ejercicios adecuados para la condición física del estudiante, ejercicios personalizados, los que consiguen una identificación asociativa con sus compañeros, una sensación de satisfacción después de la pérdida de peso, aquellos que tienen un carácter lúdicorecreativo, y aquellos que sirven para mantenerse en forma favoreciendo la salud (Rodríguez-Romo, Boned-Pascual, \& Garrido-Muñoz, 2009).

En términos generales, los resultados de la mayoría de las investigaciones se apoyan en los principios de la teoría de la autodeterminación. Deci y Ryan (2009) sugieren que la motivación no es un fenómeno único sino que varía desde niveles muy bajos de motivación a niveles muy altos. Distinguen entre diferentes tipos de motivación basados en las diferentes razones u objetivos que dan lugar a una acción. La distinción más básica es entre la motivación intrínseca, que se refiere a hacer algo porque es intrínsecamente interesante o agradable, y la motivación extrínseca, que se refiere a hacer algo por una recompensa. Las personas se involucran o no libremente en la realización de actividades, teniendo 
en cuenta una serie de mecanismos psicológicos reguladores de la conducta y buscando en la medida de lo posible una mayor orientación hacia la motivación autodeterminada (Moreno-Murcia, \& MartínezCamacho, 2006). Se asume que la persona es activa por naturaleza, con una tendencia innata a implicarse en el entorno, asimilar nuevos conocimientos y desarrollar una autorregulación autónoma (Reeve, Deci, \& Ryan, 2004; Reeve, Ryan, Deci, \& Jang, 2008). Más de tres décadas de investigación han demostrado que la calidad de la experiencia y el rendimiento pueden ser muy diferentes cuando uno se está comportando por razones intrínsecas o extrínsecas. Así pues, niveles más altos en motivación intrínseca, especialmente la motivación intrínseca hacia el logro, y en motivación extrínseca introyectada predisponen a la práctica del ejercicio físico (Granero-Gallegos, \& Baena-Extremera, 2014)

También desde la teoría de la orientación hacia la meta se han asociado la obtención de los mejores resultados con mayores expectativas y motivaciones (Valle et al., 2010). En el contexto de la educación física se ha puesto de manifiesto la relación entre las orientaciones de meta y las actitudes, la intención, la participación y el nivel de práctica de actividad física dentro y fuera de la escuela (Ruiz, \& Piéron, 2013). Por tanto, la motivación resulta un correlato importante para la consecución de hábitos de actividad física de moderada a vigorosa en adolescentes tanto en las clases de educación física como durante el tiempo libre(Owen, Astell-Burt, \& Lonsdale, 2013; Owen, Smith, Lubans, Ng, \& Lonsdale, 2014).En concreto, el profesorado y las experiencias en educación física, aunque no están presentes en el contexto de la actividadfísica en el tiempo libre, jueganun papel igual deimportante(Cañabate, Torralba, Cachón, \& Zagalaz, 2014; Gutiérrez, 2014; McDavid, Cox, \& Amorose, 2012).

Sin embargo, la familia juega un papel modesto aunque importante en la motivación hacía la práctica de la actividad física en el tiempo libre de los hijos adolescentes. En el estudio de Martins, Marques, Sarmento, y Da Costa (2015), se señalan los principales facilitadores y factores que obstaculizan la participación en actividad física, entre los que cabe destacar: actitud hacia la actividad física, motivación, percepción de la imagen corporal, diversión, influencia de amigos, familiares y profesores de educación física, y oportunidades de realizar actividad física en un contexto natural.

Para hacer frente a estas barreras intrapersonales, socio-ambientales, y físico-ambientales se debe focalizar el diseño de programas de promoción y prevención de la actividad física en poblaciones concretas (Dwyer et al., 2006).De ahí que nuestro principal objetivo sea validar un instrumento que permita medir de manera eficaz cuáles son las motivaciones de los adolescentes hacia la práctica de la actividad física y comprobar su afectación por determinadas variables sociodemográficas.

\section{Metodología}

\section{Participantes}

La muestra estuvo conformada por 852 alumnos de Educación Secundaria Obligatoria (ESO) y Bachillerato de centros públicos en la Comunidad Autónoma de Galicia, seleccionados mediante muestreo aleatorio y probabilístico. Así, un 48.9\% de los adolescentes encuestados son mujeres y un $51.1 \%$ son hombres. Sus edades oscilaron entre 12 y 17 años de edad $(M=14.86 ; D T=1.67)$. Además la distribución de los estudiantes fue similar para los ciclos: 274 alumnos de primer ciclo de ESO (32.2\%), 328 alumnos de segundo ciclo de ESO (38.5\%), y 250 alumnos del ciclo de Bachillerato (29.3\%).

\section{Instrumentos}

El instrumento utilizado fue el cuestionario Autionforme de Motivos para la Práctica de Ejercicio Físico (AMPEF), escala adaptada al español por Capdevila, Niñerola y Pintanel (2004) a partir del Exercise Motivations Inventory-2 (EMI-2) de Markland e Ingledew (1997). Dicho cuestionario está encabezado por el enunciado «personalmente practico (o practicaría) ejercicio físico» y consta de cuarenta y ocho ítems agrupados en once factores: peso e imagen corporal (siete ítems), diversión y bienestar (seis ítems), prevención y salud positiva (seis ítems), competición (cuatro ítems), afiliación (cuatro ítems), fuerza y resistencia muscular (cuatroítems), reconocimiento social (cuatro ítems), control del estrés (tres ítems), agilidad y flexibilidad (tres ítems), desafío (cuatro ítems), y urgencias de salud (tres ítems). Su formato de respuesta es tipo Likert de cero (nada verdadero para mí) a diez (totalmente verdadero para mí).

Además, el alumnado completó un cuestionario ad hoc para medir las variables sociodemográficas (edad, género y curso).

\section{Procedimiento}

El cuestionario se administró de manera colectiva en estudiantes de educación secundaria obligatoria en horario regular de clases, durante el curso 2016-17, previa autorización para su utilización con fines académicos e investigativos. Después de comunicar las instrucciones oportunas y previo consentimiento informado (centro y familias), todo el alumnado cumplimentó de forma voluntaria la información solicitada de manera individual en su grupo-clase. Se cumplieron los protocolos éticos de investigación con especial énfasis en la confidencialidad.

\section{Análisis estadístico de los datos}

Para el análisis estadístico y psicométrico de los datos se emplea el programa SPSS v. 23 y el paquete AMOS v. 23. Se llevó a cabo, en primer lugar, un análisis descriptivo de los ítems, media, desviación típica, correlación elemento-total corregida, Alpha de Cronbach si se elimina un elemento, así como los índices de asimetría y curtosis para evaluar el comportamiento normal de las variables.

A continuación, se realizó un análisis factorial exploratorio (AFE) (qualimax), con el fin de comprobar la estructura factorial inicial, se correlacionaron los factores obtenidos y se lleva a cabo un análisis factorial confirmatorio (AFC) (método de estimación de mínimos cuadrados no ponderados), para el modelo de ocho factores. En este análisis de modelos se verifica el ajuste analizando los índices básicos recomendados en la literatura (Arbuckle, 2009; Schweizer, 2010): el índice $\mathrm{x}^{2} / \mathrm{df}$ cuyos valores entre uno y dos son considerados excelentes, entre dos y tres buenos y aceptables entre tres y cinco (muestras grandes); el goodness-of-fit (GFI) y el adjusted goodness-of-fit (AGFI), con valores adecuados si son superiores a .90; el comparative fit index (CFI) que con valor superior a .90 indica un buen ajuste del modelo; y el rootsquare error of aproximation (RMSEA) que con valores inferiores a .05 son indicativos de un buen ajuste, aunque en muestras grandes son aceptados valores inferiores a 08 .

Finalmente, se valoró la consistencia interna de los factores, mediante el estadístico alfa de Cronbach. Además, se realiza un análisis inferencial a través de las puntuaciones medias y desviación típica, análisis de varianza (Anova), y comparaciones múltiples a posteriori (Prueba Scheffé). También se calculó el tamaño del efecto (d de Cohen): Valores entre . 2 y .3 indican un efecto pequeño, alrededor de .5 un efecto mediano y mayores que .8 un efecto alto.

\section{Resultados}

\section{Estadísticos descriptivos: análisis de la calidad psicométrica de} losítems

Con el fin de evaluar la distribución de la normalidad en los reactivos del cuestionario, se llevó a cabo un análisis de los ítems, mostrando medias homogéneas con desviaciones típicas alejadas de cero, lo cual indica una discriminación de los mismos. Asimismo, muestran correlaciones ítem-total corregidas positivas y superiores a .30 (valores entre .305 y .727), indicando que todos contribuyen a calcular lo que mide el test y en la misma dirección. Ello también es refrendado por el análisis del Alfa de Cronbach si se elimina un elemento, evidenciando que la supresión de cualquiera de los ítems no mejoraría la fiabilidad del conjunto del cuestionario $(\alpha=.962)$. Finalmente, la asimetría y curtosis presentan valores muy bajos, cumpliendo los criterios de normalidad. En consecuencia, los ítems que conforman el cuestionario ABPEF revelan un comportamiento normal en todos sus reactivos (Tabla 1). 
Tabla 1.

\begin{tabular}{|c|c|c|c|c|c|c|}
\hline ITEMS & M & DT & $R_{i-t}$ & $\begin{array}{c}\alpha \text { SI SE } \\
\text { ELIMINA } \\
\text { EL ÍTEM }\end{array}$ & $\begin{array}{c}\mathrm{A} . \\
(\mathrm{ET}=.132)\end{array}$ & $\begin{array}{c}\mathrm{C} . \\
(\mathrm{ET}=.263)\end{array}$ \\
\hline 1. Para mantenerme delgado & 3.70 & 2.14 & .305 & .962 & .169 & -1.306 \\
\hline 2. Para mantenerme sano & 4.90 & 1.76 & .586 & .962 & -.602 & -.413 \\
\hline 3. Porque me hace sentir bien & 4.97 & 1.88 & .618 & .961 & -.690 & -.529 \\
\hline 4. Para demostrarle a los demás lo que valgo & 2.83 & 1.98 & .539 & .962 & .799 & -.628 \\
\hline 5. Para tener un cuerpo sano & 4.87 & 1.75 & .655 & .961 & -.524 & -.588 \\
\hline 6. Para tener más fuerza & 4.33 & 2.15 & .675 & .961 & -.247 & -1.278 \\
\hline 7. Porque me gusta la sensación que tengo al hacer ejercicio & 4.73 & 2.05 & .650 & .961 & -.579 & -.924 \\
\hline 8. Para pasar el tiempo con los amigos & 4.12 & 2.12 & .491 & .962 & -.149 & -1.270 \\
\hline 9. Porque el médico me ha aconsejado hacer ejercicio & 2.38 & 1.86 & .344 & .962 & 1.175 & .178 \\
\hline 10. Porque me gusta intentar ganar cuando hago ejercicio & 3.25 & 2.21 & .519 & .962 & .454 & -1.250 \\
\hline $\begin{array}{l}\text { 11. Para estar más ágil } \\
\text { 1. }\end{array}$ & 4.39 & 1.91 & .663 & .961 & -.343 & -.909 \\
\hline 12. Para tener unas metas por las que esforzarme & 4.14 & 2.11 & .674 & .961 & -.135 & -1.300 \\
\hline 13. Para perder peso & 3.68 & 2.29 & .427 & .962 & .168 & -1.491 \\
\hline 14. Para evitar problemas de salud & 4.26 & 2.10 & .568 & .962 & -.248 & -1.224 \\
\hline 15. Porque el ejercicio me da energía & 4.11 & 2.11 & 608 & .961 & -160 & -1.294 \\
\hline 16. Para tener buen cuerpo & 4.25 & 2.15 & .551 & .962 &,- 217 & -1.311 \\
\hline 17. Para comparar mis habilidades con las de los demás & 2.46 & 1.92 & .533 & .962 & 1.138 & .057 \\
\hline 18. Porque ayuda a reducir la tensión & 3.44 & 2.14 & .547 & .962 & .305 & -1.267 \\
\hline 19. Porque quiero disfrutar de buena salud & 4.46 & 1.97 & .618 & .961 & -.350 & -.954 \\
\hline 20. Para aumentar mi resistencia & 4.50 & 2.03 & .698 & .961 & -.430 & -1.035 \\
\hline 21. Porque el ejercicio hace que me sienta satisfecho & 4.31 & 2.07 & 631 & .961 & -.226 & -1.196 \\
\hline 22. Para disfrutar de los aspectos sociales del ejercicio & 3.56 & 2.08 & .622 & .961 & .239 & -1.234 \\
\hline 23. Para evitar una enfermedad que se da mucho en mi familia & 2.45 & 2.05 & .344 & .962 & 1.158 & -.148 \\
\hline 24. Porque me lo paso bien compitiendo & 3.78 & 2.21 & .579 & .962 & .094 & -1.395 \\
\hline $\begin{array}{l}\text { 25. Para mantener la flexibilidad } \\
\text { 25ituo }\end{array}$ & 3.56 & 2.07 & .563 & .962 & .269 & -1.181 \\
\hline 26. Para tener retos que superar & 3.89 & 2.22 & .670 & .961 & .029 & -1.418 \\
\hline 27. Para controlar mi peso & 3.75 & 2.22 & .536 & .962 & .121 & -1.405 \\
\hline 28. Para evitar problemas cardiacos & 3.29 & 2.22 & .571 & .962 & .431 & -1.282 \\
\hline 29. Para mejorar mi aspecto. & 3.83 & 2.17 & .577 & .962 & .058 & -1.361 \\
\hline 30. Para obtener reconocimiento cuando me supero & 3.39 & 2.16 & 669 & .961 & .336 & -1.297 \\
\hline 31. Para ayudarme a superar el estrés & 3.89 & 2.23 & .551 & .962 & .022 & -1.430 \\
\hline 32. Para sentirme más sano & 4.74 & 2.06 & .650 & .961 & -.551 & -.970 \\
\hline 33. Para ser más fuerte & 4.02 & 2.14 & 675 & .961 & -.021 & -1.309 \\
\hline 34. Porque el ejercicio me produce diversión & 4.70 & 2.00 & .631 & .961 & -.472 & -.963 \\
\hline 35. Para divertirme haciendo ejercicio con otras personas & 4.34 & 2.13 & .525 & .962 & -.318 & -1.209 \\
\hline 36. Para recuperarme de una enfermedad/lesión & 2.69 & 2.06 & .442 & .962 & .884 & -.632 \\
\hline 37. Porque disfruto haciendo competición física & 3.80 & 2.14 & .632 & .961 & .098 & -1.316 \\
\hline 38. Para tener más flexibilidad & 3.58 & 2.10 & .553 & .962 & .260 & -1.223 \\
\hline 39. Para desarrollar mis habilidades personales & 3.94 & 2.09 & .727 & .961 & -.026 & -1.286 \\
\hline 40. Para quemar calorías & 4.07 & 2.19 & .564 & .962 & -.078 & -1.376 \\
\hline 41. Para estar más atractivo & 3.49 & 2.19 & .510 & .962 & .331 & -1.313 \\
\hline $\begin{array}{l}\text { 42. Para conseguir hacer cosas que los demás no pueden hacer } \\
\text { dense }\end{array}$ & 2.97 & 2.09 & .580 & .962 & .620 & -.998 \\
\hline 43. Para liberar la tensión & 4.01 & 2.23 & .601 & .961 & -.047 & -1.420 \\
\hline 44. Para desarrollar mi mis músculos & 3.88 & 2.17 & .710 & .961 & .053 & -1.364 \\
\hline 45. Porque haciendo ejercicio me siento muy bien & 4.68 & 2.09 & .674 & .961 & -.458 & -1.088 \\
\hline 46. Para hacer amigos & 3.39 & 2.19 & .499 & .962 & .348 & -1.307 \\
\hline 47. Porque me divierte hacer ejercicio sobre todo si & 3.73 & 2.32 & .584 & .962 & .132 & -1.520 \\
\hline 48. Para probarme a mi mismo & 4.20 & 2.18 & .666 & .961 & -.184 & -1.344 \\
\hline
\end{tabular}

mediante el coeficiente de correlación de Pearson del cuestionario AMPEF. Se observa, a nivel global, que todas las correlaciones fueron positivas y significativas $(p<.01)$, apreciando una mayor magnitud entre afiliación, diversión, bienestar y competición, reconocimiento social, desafío $(r=.74)$, y entre fuerza, resistencia muscular y competición, reconocimiento social, desafío $(r=.70)$. Por otro lado, las de menor magnitud fueron encontradas entre: urgencias de salud y fuerza, resistencia muscular $(r=.41)$; urgencias de salud y afiliación, diversión, bienestar $(r=.41)$; urgencias de salud y peso, imagen corporal ( $r=.41)$; y, afiliación, diversión, bienestar y peso, imagen corporal $(r=.30)$.

En último lugar, el análisis factorial confirmatorio (método de estimación por máxima verosimilitud) de la versión del cuestionario de 48 ítems y ocho factores presenta resultados satisfactorios (Figura 1). Así, las cargas factoriales en los ocho factores oscilan entre .47y .84 (consideradas buenas) y las saturaciones factoriales estandarizadas se encuentran entre moderadas (.40) y altas (.71), excepto en los ítems 23 (.27), 8 (.28), 9 (.28), 46 (.30), 4 (.31), 17 (.34), 36 (.37), y 38 (.38) que se consideran bajas.

Finalmente, se pone a prueba la hipótesis de ocho factores. Los resultados evidencian que la mayoría de los estimadores están dentro del rango esperado o muy cercano a él, lo cual indica un ajuste aceptable: $\mathrm{x}^{2} / \mathrm{df}=2.635$, $\mathrm{GFI}=.909, \mathrm{AGFI}=.917, \mathrm{CFI}=.901, \mathrm{RMSEA}=.047$ $(\mathrm{IC} 90 \%=.046-.052)$.

\section{Confiabilidad del cuestionario}

El cuestionario considerado en su totalidad presen-

Tabla 2.

Propiedades psicométricas del AMPEF

Con la finalidad de obtener evidencia sobre la dimensionalidad del cuestionario, se llevó a cabo, en primer lugar un análisis factorial exploratorio. Previa- AMPEF32 mente al mismo, se comprueba que los índices de Kai- $\begin{aligned} & \text { AMPEF19 } \\ & \text { AMPEF14 }\end{aligned}$ $\begin{array}{lll}\text { mente al mismo, se comprueba que los indices de Kai- } & \text { AMPEF14 } & .654 \\ \text { ser-Meyer-Olkin (KMO) y de esfericidad de Bartlett } & \text { AMPEF3 } & .551 \\ \text { AMPEF21 } & .538\end{array}$ son adecuados para ejecutar el AFE (KMO = .959; ${ }_{\text {AMPEF15 }}$ Bartlett $=27844.57 ; p<.001)$. Así pues, se realizó el AMPEF24 AFE por el Método de Componentes Principales, con AMPEF10 una rotación quartimax (recogiendo únicamente los pe- AMPEF26 Inatación quartion (recogiendoúnicamentelos pe- AMPEF2 sos superiores a .30). Del análisis realizado se extraje- AMPEF12 ron un total de ocho factores que explican un 65.24\% de AMPEF48 la varianza total de los datos: factor 1, Prevención y AMPEF17 salud positiva (P/SP) con pesos factoriales entre .52 y AMPEF4 .71 (Ej. «Para mantenerme sano»); factor 2, Competi- AMPEF1 ción/Reconocimiento Social/Desafío(C/RS/D) con pe- AMPEF27 AMPEF40 sos factoriales situados entre .43 y .72(Ej. «Porque me AMPEF41 gusta intentar ganar cuando hago ejercicio»); factor 3, AMPEF29 Peso e Imagen Corporal (P/IC) con pesos factoriales AMPEF35 que van de .65 a .74 (Ej. «Para perder peso»); factor 4, AMPEF46 Afiliación, diversión y bienestar (A/D/B), con pesOS AMPEF34 factoriales que oscilan entre .50y .74 (Ej. «Para disfru- $\begin{array}{ll}\text { AMPEF7 } \\ \text { AMPEF22 }\end{array}$ tar de los aspectos sociales del ejercicio»); factor 5, AMPEF43 Control del Estrés (CE), con pesos factoriales en este AMPEF18 factor oscilan entre .63 y .70 (Ej. «Para liberar la ten- ${ }_{\text {AMPEF33 }}$ sión»); factor 6, Fuerza y Resistencia Muscular (F/ AMPEF44 $\mathrm{RM}$ ) con pesos factoriales que oscilan entre .68 y .73 AMPEF20 RM) con pesos factoriales que oscilan entre .68 y .73 AMPEF23 (Ej. «Para tener más fuerza»); factor 7, Urgencias de AMPEF36 Salud(US), con pesos factoriales que oscilan entre .53 AMPEF28 y .56 (Ej. «Porque el médico me ha aconsejado hacer AMPEF38 ejercicio»); y, el factor 8, Agilidad y Flexibilidad(A/F), con pesos factoriales entre .59 y .78 (Ej. «Para mantener flexibilidad»)(Tabla 2).

A continuación, se analizó la validez convergente

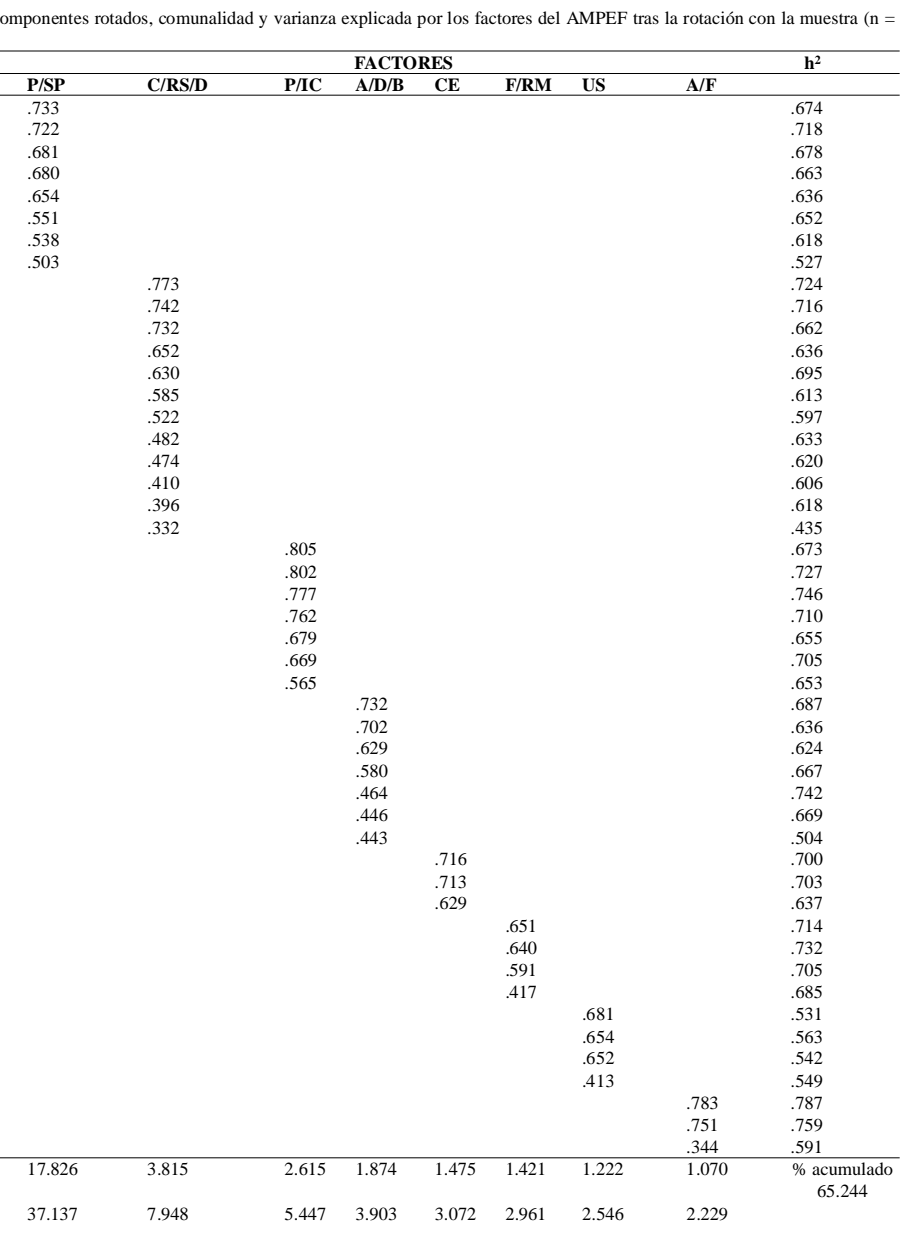




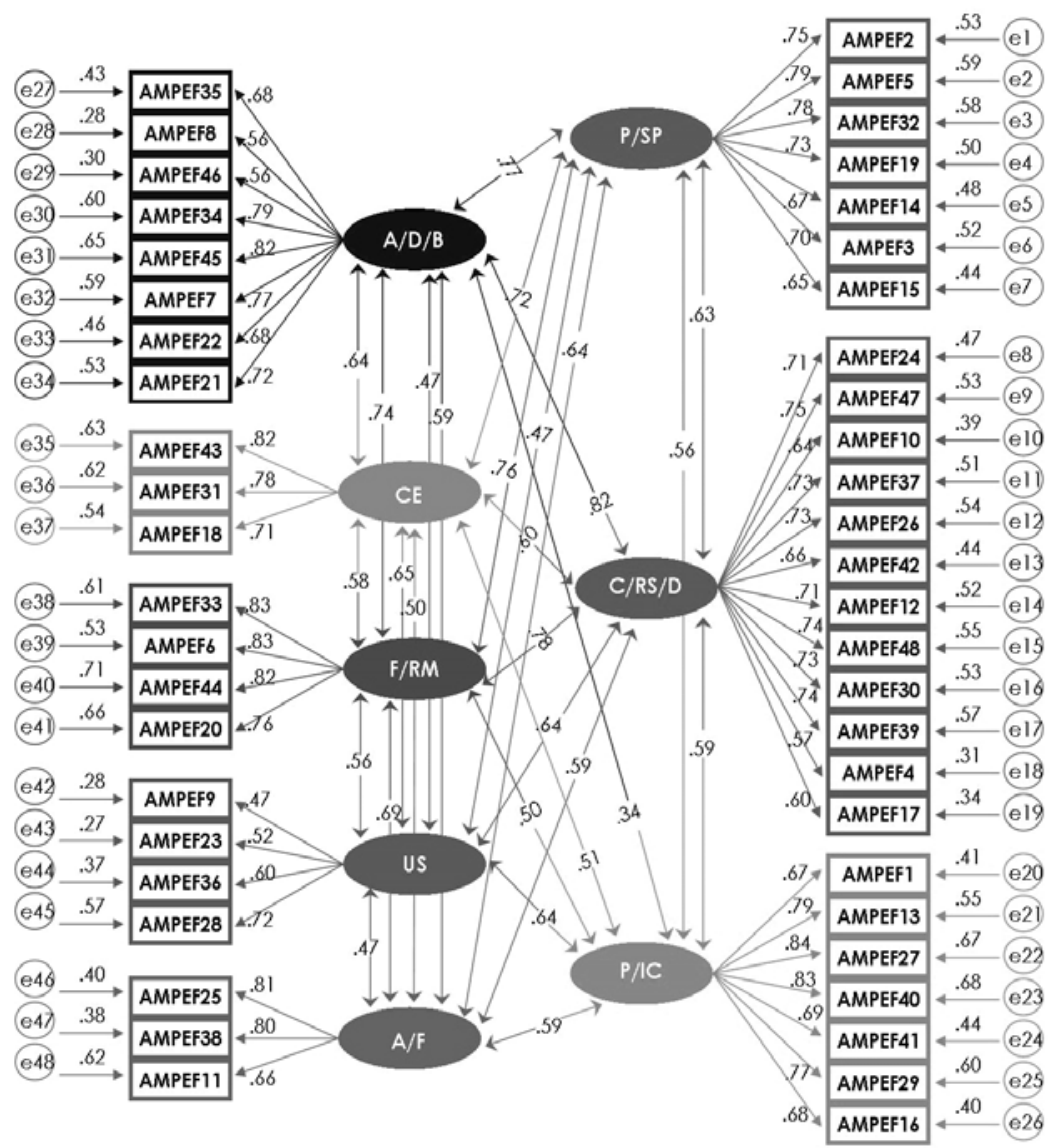

Figura 1.

Coeficientes de regresión y saturaciones factoriales estandarizadas del cuestionario AMPEF (M8F)

$p<.05$ ), siendo mejor en el género masculino (tamaño del efecto pequeño, $d=.15$ ).

Atendiendo a la variable edad, muestra diferencias significativas en Urgencias de salud $\left(F_{(2,849)}=6.56 ; p<.01\right)$, confirmando la prueba Scheffé que se producen entre los que tienen 12 o 13 años con los que tienen entre 14 y 18 años (tamaño del efecto pequeño, $d=.27$ y .25 ). Si se hace referencia al ciclo académico, las diferencias significativas se aprecian en el control del estrés $\left(F_{(2,849)}=4.66 ; p<.05\right)$, confirmando la prueba Scheffé que se producen entre los que cursan Bachillerato o $2^{\circ}$ ciclo de ESO con los que lo hacen en $1^{\circ}$ de laESO (tamaño del efecto pequeño, $d=.27$ y .13); y en las Urgencias de salud $\left(F_{(2849)}=11.83 ; p<.01\right)$, confirmando la prueba Scheffé que se producen entre los que cursan $1^{\circ}$ ciclo de ESO con los que lo hacen en Bachillerato (tamaño del efecto medio, $d=.37$ ).

Finalmente destacar que los factores prevención/salud positiva, peso e imagen corporal y agilidad/flexibilidad no resultan significativos en ninguna de las tres variables sociodemográficas (género, edad y ciclo escolar).

\section{Discusión}

La finalidad de esta investigación ha sido comprobar las propiedades psicométricas del autoinforme de motivación para la práctica del ejercicio físico (AMPEF), así como analizar las diferencias de motivación según género, edady ciclo escolar, en el alumnado de educación secundaria obligatoria y bachillerato. La impor-

ta un índice de fiabilidad muy alto $(\alpha=.962)$. Los factores ofrecen índices ligeramente inferiores, pero muy similares entre ellos (P/SP = $.882 ; \mathrm{C} / \mathrm{RS} / \mathrm{D}=.918 ; \mathrm{P} / \mathrm{IC}=.898 ; \mathrm{A} / \mathrm{D} / \mathrm{B}=.884 ; \mathrm{CS}=.813 ; \mathrm{F} / \mathrm{RM}=$ .883; $\mathrm{US}=.787 ; \mathrm{A} / \mathrm{F}=.789$ ).

\section{Efectos de interacción del género, edad y ciclo escolar}

Para analizar la existencia de diferencias significativas se han realizado análisis de varianza (ANOVA). Así pues, se han recogidoúnicamente los factores que presentan diferencias significativas (Tabla 3).

En referencia al género, presenta diferencias significativas en Competición/Reconocimiento Social/Desafío $\left(F_{(1,850)}=38.6 ; p<.01\right)$, siendo

Tabla 3.

Estadísticos descriptivos y análisis de las diferencias en función del género, edad y ciclo académico

\begin{tabular}{|c|c|c|c|c|c|c|}
\hline \multicolumn{2}{|l|}{ FACTOR del AMPEF } & $\mathbf{M}$ & DT & $\mathbf{F} / \mathbf{p}$ & Scheffé & d Cohen \\
\hline \multicolumn{7}{|c|}{ GÉNERO } \\
\hline \multirow{2}{*}{$\begin{array}{l}\text { Competición/Reconocimiento } \\
\text { Social/Desafío }\end{array}$} & Masculino & 48.04 & 16.23 & 38.6 & \multirow{2}{*}{$>$ masculino } & \multirow{2}{*}{.43} \\
\hline & Femenino & 41.03 & 16.65 & $<.01$ & & \\
\hline \multirow{2}{*}{ Afiliación/Diversión/Bienestar } & Masculino & 37.07 & 12.44 & 27.9 & \multirow[t]{2}{*}{$>$ masculino } & \multirow{2}{*}{.36} \\
\hline & ${ }^{\mathrm{r}}$ Femenino & 32.45 & 13.02 & $<.01$ & & \\
\hline \multirow{2}{*}{ Fuerza/Resistencia Muscular } & Masculino & 18.22 & 7.08 & 25.4 & \multirow{2}{*}{$>$ masculino } & \multirow{2}{*}{.37} \\
\hline & Femenino & 15.64 & 7.04 & $<.01$ & & \\
\hline \multirow{2}{*}{ Urgencias de Salud } & Masculino & 10.78 & 7.14 & 4.9 & \multirow{2}{*}{$>$ masculino } & \multirow[b]{2}{*}{.15} \\
\hline & Femenino & 9.81 & 5.54 & $<.05$ & & \\
\hline \multicolumn{7}{|c|}{ EDAD } \\
\hline \multirow{3}{*}{ Urgencias de Salud } & $12-13$ & 11.63 & 8.24 & & & \\
\hline & $14-15$ & 9.71 & 5.66 & \multirow{2}{*}{$\begin{array}{l}<.01 \\
<.01\end{array}$} & $12-13 / 14-15$ & .27 \\
\hline & $16-18$ & 9.92 & 5.47 & & $12-13 / 16-18$ & .25 \\
\hline \multicolumn{7}{|c|}{ CICLO ACADÉMICO } \\
\hline \multirow{3}{*}{ Control del Estrés } & $1^{\circ} \mathrm{ciclo}$ ESO & 10.36 & 5.46 & \multirow{3}{*}{$\begin{array}{l}4.66 \\
<.05\end{array}$} & \multirow{3}{*}{$\begin{array}{c}\mathrm{Bach} / 1^{\circ} \mathrm{ESO} \\
2^{\circ} \mathrm{ESO} / 1^{\circ} \mathrm{ESO}\end{array}$} & \multirow{3}{*}{$\begin{array}{l}.27 \\
.13\end{array}$} \\
\hline & $2^{\circ}$ ciclo ESO & 11.06 & 5.70 & & & \\
\hline & Bachiller & 11.85 & 5.43 & & & \\
\hline \multirow{3}{*}{ Urgencias de Salud } & $1^{\circ}$ ciclo ESO & 11.79 & 7.89 & \multirow{3}{*}{$\begin{array}{l}11.83 \\
<.01\end{array}$} & \multirow{3}{*}{$1^{\circ} \mathrm{ESO} / \mathrm{Bach}$} & \multirow{3}{*}{.37} \\
\hline & $22^{\circ}$ ciclo ESO & 9.75 & 5.73 & & & \\
\hline & Bachiller & 9.34 & 4.93 & & & \\
\hline
\end{tabular}

mejor en el género masculino (tamaño del efecto mediano $d=.43$ ); Afiliación/Diversión/Bienestar $\left(F_{(1,850)}=27.9 ; p<.01\right)$, siendo mejor en el género masculino (tamaño del efecto mediano $d=.36$ ); Fuerza/Resistenciamuscular $\left(F_{(1,850)}=25.4 ; p<.01\right)$, siendo mejor en el género masculino (tamaño del efecto mediano, $d=.37)$; y, Urgencias de $\operatorname{salud}\left(F_{(1,850)}=4.9\right.$; tancia del mismo radica en que la motivación parece ser la pieza clave en la elección de determinadas actividades y en las causas que determinan lapermanencia o abandono de la misma (Moreno, Cervelló, \& Martínez, 2007). Además se centra en la etapa de la adolescencia, periodo de vida que está originando enorme interés en cuanto al análisis de estilos de vida saludables ya que es en esta etapa vital donde el alumnado suele abandonar las prácticas de actividad física regular (Plaza, Jové, Hernández, \& Reverter, 2013; Van der Horst, Paw, Twisk, \& Van Mechelen, 2007).

Así pues, el estudio examina las propiedades psicométricas del AMPEF, evidenciando precisión, estabilidad y puede ser generalizable a otras poblaciones adolescentes en la medición de la motivación hacia la práctica del ejercicio físico. Los hallazgos referidos dan cuenta de que los ítems que conforman el autoinforme AMPEF, revelan un comportamiento normal en todos sus reactivos, al mostrar índices de asimetría y curtosis inferiores a dos, por lo que cumplen con el supuesto de normalidad (Bollen, \& Long, 1993). Además, todos los ítems (método ítemescala) poseen niveles de discriminación superiores al mínimo requerido de .20 (Kline, 2005), lo cual califica el nivel de discriminación bueno y confirma que los ítems que conforman las dimensiones miden la motivación para la práctica del ejercicio físico.

En relación a la estructura factorial obtenida se ponen de manifiesto ocho factores (Prevención/Salud Positiva, Competición/Reconocimiento Social/Desafío,Peso/Imagen Corporal,Afiliación/Diversión/Bienestar, Control de Estrés, Fuerza/Resistencia Muscular, Urgencias de Salud, Agilidad/Flexibilidad) que explican el 65.24\% de la varianza. La distribución de los ítems en los factores fue muy similar a la encontrada por Capdevila et al.(2004). No obstante, la particularidad de la población de este estudio, puede explicar la variabilidad en los factores, siendo coincidente en prevención y salud positiva, peso e imagen corporal, control del estrés, fuerza y resistencia muscular, urgencias de salud, y agilidad/ flexibilidad, pero diferenciándose en la unión de los factores competición/reconocimiento social/desafío, y afiliación/diversión y bienestar. 
Por lo que respecta a la validez de criterio obtenida al correlacionar las ocho dimensiones en la motivación para el ejercicio físico, mediante la prueba de correlación de Pearson, identifica una correlación significativa ( $p<.001)$, positiva y de grado medio-alto, en la misma línea que el estudio de Capdevila et al. (2004). A su vez, el Análisis Factorial Confirmatorio realizado para conocer la validez de constructo, mostró que este autoinforme mantiene las ocho dimensiones del Análisis Factorial Exploratorio y que los índices obtenidos para valorar la bondad de ajuste en este estudio fueron adecuados.

Finalmente, en la confiabilidad en términos de consistencia interna, mediante el coeficiente de Alfa de Cronbach, se aprecia que las ocho dimensiones cuentan con valores que van desde .78 a .91 , y para la totalidad de la escala cuenta con un valor de .96, lo que califica a la coherencia de la escala como muy buena y aceptable en la población investigada. Estos datos coinciden con los obtenidos en la versión original de Capdevila et al. (2004).

Por otra parte, en el estudio descriptivo-inferencial se analizaron las dimensiones obtenidas en la escala demotivación para la práctica del ejercicio físico según género, edad y ciclo educativo. En un primer momento, destacar que el factor urgencias de salud es el único que ha resultado significativo en las tres variables analizadas, lo cual revela la importancia que los adolescentes otorgan a dicho motivo para la realización del ejercicio físico, corroborando hallazgos anteriores (Castañeda, \& Campos, 2012; García-Ferrando, 2006; Moreno, Cervelló, Borges, \& Conte, 2009). En cuanto al género, los resultados dan cuenta de que el masculino presenta mejor motivación en competición/reconocimiento social/desafío, afiliación/diversión/bienestar, fuerza/resistencia muscular, y urgencias de salud, hacia la práctica del ejercicio físico. Asimismo, ambos géneros perciben por igual prevención/salud positiva, peso e imagen personal, control de estrés y agilidad/flexibilidad. Estos datos refuerzan los trabajos de diferentes investigaciones (Rodríguez, Morera, Barrantes, \& Ugalde, 2014; Wilson, \& Rodgers, 2002) que también coinciden en señalar como motivos más importantes en los hombres, la competición, fuerza, resistencia y reconocimiento social, mientras que las mujeres se inclinan por la imagen corporal y estética. Dichos resultados están en la línea del estudio realizado por Isorna, Rial y VaqueroCristóbal (2014) al indicar que los chicos adolescentes práctican más deporte que las chicas. Por último, la edad solamente presenta diferencias en urgencias de salud, apreciando que los adolescentes más jóvenes (12-13 años) tienen una mejor motivación hacia la práctica del ejercicio físico (Carcamo-Oyarzon, Wydra, Hernandez-Mosqueira, \& MartinezSalazar, 2017). Estudios con rangos más amplios de edad, encontraron que los individuos más jóvenes tenían mayor inclinación hacia aspectos competitivos, frente a motivos más lúdicos, estéticos o vergüenza a edades más avanzadas (Oldman, Daley, \& Richard, 2004). De modo similar, el ciclo académico muestra que en el control del estrés la motivación es favorable a los ciclos superiores ( $2^{\circ}$ ciclo y bachiller), mientras que las urgencias de salud al primer ciclo.

\section{Conclusiones}

El presente estudio supone, por tanto, una aportación teórica y práctica al estudio y tratamiento de la motivación hacia la práctica del ejercicio físico como otras aportaciones anteriores (Moreno-Murcia, de San Román Blanco, Martínez-Galindo, Villodre \& González-Cutre, 2006). No obstante presenta limitaciones como la selección aleatoria de la muestra acotando edades y regiones, o la afectación por sesgo en los resultados por el falseamiento o la deseabilidad social en las medidas de autoinforme. Por tanto, será importante poder identificar las motivaciones de los adolescentes y poner en marcha programas de promoción del ejercicio físico (Ferreira, \& Naja, 2005), en aras de conseguir una mayor adherencia a la práctica físico-deportiva (Moreno-Murcia, Borges, Marcos, Sierra, \& Huéscar, 2012).

\section{Agradecimientos}

Soporte financiero: El trabajo ha sido realizado al amparo del proyecto «Aprender en movimiento» (Ref. 15VI05) de la Universidad de Vigo.

\section{Referencias}

Arbuckle, J.L. (2009). Amos18 User's Guide. Chicago: SPSS.

Atkin, A.J., Adams, E., Bull, F.C., \& Biddle S.J.H. (2012). Nonoccupational sitting and mental well-being in employed adults. Annals of Behavioral Medicine, 43, 181-188. doi: 10.1007/s12160011-9320-y

Bollen, K.A., \& Long, J.S. (1993). Testing Structural Equation Models. Sage: Newbury Park, CA.

Cañabate, D., Torralba, J.P., Cachón, J., \& Zagalaz, Mª L. (2014). Perfiles motivacionales en las sesiones de educación física. Retos. Nuevas tendencias en Educación Física, Deporte y Recreación, 26, 34-39.

Capdevila, Ll., Niñerola, J., \& Pintanel, M. (2004). Motivación y actividad física: el autoinforme de motivos para la práctica de ejercicio físico (AMPEF). Revista de Psicología del Deporte, 13(1), 55-74.

Carcamo-Oyarzun, J., Wydra, G., Hernandez-Mosqueira, C., \& Martinez-Salazar C. (2017). Actitudes hacia la educación física: Grados de importancia y conformidad según escolares de Chile y Alemania. Una mirada intercultural. Retos, 32, 158-162.

Castañeda, C., \& Campos, $M^{\mathrm{a}}$ C. (2012). Motivación de los estudiantes de la Facultad de Ciencias de la Educación (Universidad de Sevilla) hacia la práctica de actividad físico-deportiva. Retos. Nuevas tendencias en Educación Física, Deporte y Recreación, 22, 5761.

Deci, E.L., \& Ryan, R.M. (2009). The «what» and «why» of goal pursuits: human needs and the self-determination of behaviour. Psychological Inquiry, 11, 227-268. doi: 10.1207/ S15327965PLI1104_01

Dwyer, J.J.M., Allison, K.R., Goldenberg, E.R., Fein, A.J., Yoshida, K.K., \& Boutilier, M.A.(2006).Adolescent girls' perceived barriers to participation in physical activity. Adolescence, 161(41), 75-89.

Ferreira, M.S., \& Naja, A.L. (2005). Programas e campanhas de promoção da atividade física. Ciência e Saude Coletiva, 10, 207219.

García-Ferrando, M. (2006). Posmodernidad y deporte: Entre la individualización y la masificación. Encuesta sobre hábitos deportivos de los españoles 2005. Madrid: CSD y CIS

Granero-Gallegos,A., \& Baena-Extremera,A. (2014). Predicción de la motivación autodeterminada según las orientaciones de meta y el clima motivacional en Educación Física. Retos. Nuevas tendencias en Educación Física, Deporte y Recreación, 25, 23-27.

Gutiérrez, M. (2014). Relaciones entre el clima motivacional, las experiencias en educación física y la motivación intrínseca de los alumnos. Retos. Nuevas tendencias en Educación Física, Deporte y Recreación, 26, 9-14.

Isorna, M., Rial, A., \& Vaquero-Cristóbal, R. (2014). Motivaciones para la práctica deportiva en escolares federados y no federados. Retos. Nuevas tendencias en Educación Física, Deporte y Recreación, 25, 80-84.

Kilpatrick, M., Sanderson, K., Blizzard, L., Teale, B., \& Venn, A. (2013). Cross-sectional associations between sitting at work and psychological distress: Reducing sitting time may benefit mental health. Mental Health and Physical Activity, 6(2), 103-109. doi: 10.1016/j.mhpa.2013.06.004

Kimberly, K.M.S., Hultquist, C.N., \& McLester, Y.R. (2013). A Comparison of Motivational Factors and Barriers to Physical Activity Among Traditional Versus Nontraditional College Students. Journal of American College Health, 61(2), 60-66. doi: 10.1080/ 07448481.2012.753890

Kline, R.B. (2005). Principles and practice of structural equation modeling (2nd Edition). New York: The Guilford Press.

Kumar, J., Adhikari, K., Li, Y.J., Lindshield, E., Muturi, N., \& Kidd, T. (2016). Identifying barriers, perceptions and motivations related to healthy eating and physical activityamong 6th to 8th grade, rural, limited-resource adolescents. Health Education, 116(2), 123-137. doi: 10.1108/HE-03-2014-0035

Mailey, E.L., Huberty, J., Dinkel, D., \& McAuley, E. (2014). Physical 
activity barriers and facilitators among working mothers and fathers. BMC Public Health, 14, 657. doi: 10.1186/1471-2458-14-657

Markland, D., \& Ingledew, D.K. (1997). The measurement of exercise motives: Factorial validity and invariance across gender of a revised Exercise Motivations Inventory. British Journal of Clinical Psychology, 2, 361-376.

Martins, J., Marques, A., Sarmento, H., \& Da Costa, F.C. (2015). Adolescents' perspectives on the barriers and facilitators of physical activity: a systematic review of qualitative studies. Health Education Research, 30(5), 742-755. doi: 10.1093/her/cyv042

McDavid, L., Cox, A.E., \& Amorose, A.J. (2012). The relative roles of physical education teachers and parents in adolescents' leisuretime physical activity motivation and behavior. Psychology of Sport and Exercise, 13(2), 99-107. doi: 10.1016/j.psychsport.2011.10.003

Moreno, J.A., Cervelló, E., Borges, F., \& Conte, L. (2009). O interesse pela opinião do praticante de exercício físico como papel importante na predição do motivo fitness/saúde. Fitness \& Performance Journal, 8(4), 247-253.

Moreno, J.A., Cervelló, E., \& Martínez, A. (2007). Validación de la Escala de Medida de los Motivos para la Actividad Física-Revisada en españoles: Diferencias por motivos de participación. Anales de psicología, 23(1), 167-176.

Moreno-Murcia, J.A., Borges, F., Marcos, P.J., Sierra,A.C., \& Huéscar, E. (2012). Motivación, frecuencia y tipo de actividad en practicantes de ejercicio físico. Revista Interrnacional de Medicina y Ciencias de la Actividad física y del Deporte, 12(48), 649-662.

Moreno-Murcia, J.A., de San Román-Blanco, M.L., Martínez-Galindo, C., Villodre, N.A., \& González-Cutre, D. (2006). Validación preliminar de la escala de percepción del clima motivacional de los iguales (CMI) y la escala de las orientaciones de meta en el ejercicio (GOES) con practicantes españoles de actividades físico-deportivas. Revista Iberoamericana de Psicología del Ejercicio y el Deporte, 1(2), 13-28.

Moreno-Murcia, J.A., \& Martínez-Camacho, A. (2006). Importancia de la Teoría de la Autodeterminación. Cuadernos de Psicología del Deporte, 6(2), 39-54.

Morente, H., Zagalaz, M., Molero, D., \& Carrillo, S. (2012). Prevención de la obesidad infantil a través de una motivación intrínseca hacia la práctica de actividad física. Retos. Nuevas Tendencias en Educación Física, Deporte y Recreación, 22, 33-37.

Nicholson, S., Sniehotta, F.F., Van Wijck, F., Greig, C.A., Johnston, M., McMurdo, M.E., Dennis, M., \& Mead, G.E. (2013). A systematic review of perceived barriers and motivators to physical activity after stroke. International Journal of Stroke, 8(5), 357-364. doi: 10.1111/j.1747-4949.2012.00880.x

Oldman, A., Daley, A., \& Richard, N. (2004). Motives for sport and exercise in younger and older half marathon runners. Journal of Sports Science, 22, 310-311.

Organización Mundial de la Salud (OMS) (2010). Recomendaciones mundiales sobre actividad fisica para la salud. World Health Organization, Geneva. 2010 Recuperado de http://apps.who.int/ iris/bitstream/10665/44441/1/9789243599977_spa.pdf

Owen, K.B., Astell-Burt, T., \& Lonsdale, C. (2013). The Relationship Between Self-Determined Motivation and Physical Activity in Adolescent Boys. Journal of Adolescent Health, 53(3), 420-422. doi: 10.1016/j.jadohealth.2013.05.007

Owen, K.B., Smith, J., Lubans, D.R., Ng, J.Y.Y., \& Lonsdale, C. (2014). Self-determined motivation and physical activity in children and adolescents: A systematic review and meta-analysis. Preventive Medicine, 67, 270-279. doi: 10.1016/j.ypmed.2014.07.033

Peeters, C., Marchand, H., Tulloch, H., Sigal, R.J., Goldfield, G.S., Hadjiyannakis, S., \& Kenny, G.P. (2012). Perceived facilitators, barriers, and changes in a randomized exercise trial for obese youth: A qualitative inquiry. Journal of Physical Activity \& Health, 9(5), 650-660. DOI: 10.1123/jpah.9.5.650

Perry, C., \& Hoffman, B. (2010). Assessing tribal youth physical activity and programming using a community-based participatory research approach. Public Health Nursing, 27(2), 104-114. doi: 10.1111/ j.1525-1446.2010.00833.x

Plaza, D., Jové, M.C., Hernández, V., \& Reverter, J. (2013). Valoración de los docentes de Educación Física de las actividades deportivas extraescolares. EmásF. Revista Digital de Educación Física, 4(21), 69-78.

Reeve, J., Deci, E., \& Ryan, R. (2004). Self-determination theory: A dialectical framework for understanding sociocultural influences on student motivation. En D. McInerney, y S. Van Etten, Big theories revisited (pp. 31-60). Greenwich: information Age.

Reeve, J., Ryan, R., Deci, E., \& Jang, H. (2008). Understanding and promoting autonomous self-regulation: A self-determination perspective. En D. H. Schunk., y B. J. Zimmerman, Motivation and self-regulated learning: Theory, research, and applications (pp. 223-244). Londres: LEA.

Rodríguez, A., Morera, M., Barrantes, K., \& Ugalde, J.A. (2014). Relación entre los Factores Motivacionales, la Edad y el Sexo en las Personas Participantes de un Proyecto de Natación. MHSalud, 11(1), 13-25.

Rodríguez-Romo, G, Boned-Pascual, C., \& Garrido-Muñoz, M. (2009). Motivos y barreras para hacer ejercicio y practicar deportes en Madrid. Revista Panamericana de Salud Pública, 26(3), 244-254. doi: 10.1590/S1020-49892009000900009

Ruiz, F., \& Piéron, M. (2013). Orientaciones de meta en Educación Física y nivel de actividad físico-deportiva en estudiantes mexicanos. Universitas Psychologica, 12(1), 235-247.

Schweizer, K. (2010). Some guidelines concerning the modeling of traits and abilities in test construction. European Journal of Psychological Assessment, 26(1), 1-2. doi: 10.1027/1015-5759/a000001

Serra, J.R. (2014). Análisis de la práctica de actividad física durante el recreo escolar en adolescentes de la provincia de Huesca. Archivos de Medicina del Deporte, 31(4), 257-262.

Trites, S.J., \& Elgar, F.J. (2010). Making time to exercise: an analysis of the impact of motivation and elective high school physical education on adolescent physical activity level. 2nd International conference on education and new learning technologies, Barcelona: 5-7 July. EDULEARN10 Proceedings. p. 5644-5652.

Valle,A., Rodríguez, S., Cabanach, R.G., Núñez, J.C., González-Pienda, J.A., \& Rosario, P. (2010). Perfiles motivacionales y diferencias en variables afectivas, motivacionales y de logro. Universitas Psychologica, 9(1), 109-121.

Van der Horst, K., Paw, M.J., Twisk, J.W., \& Van Mechelen, W. (2007). A brief review on correlates of physical activity and sedentariness in youth. Medicine \& Science in Sports \& Exercise, 39(8), 1241-1250. doi: 10.1249/mss.0b013e318059bf35

Van Uffelen, J.G, Van Gellecum, Y.R., Burton, N.W., Peeters, G, Heesch, K.C., \& Brown W.J. (2013). Sitting-time, physical activity, and depressive symptoms in mid-aged women. American Journal of Preventive Medicine, 45(3), 276-281. doi: 10.1016/ j.amepre.2013.04.009

Vergara, F.V., Santibáñez, M.A., Herrera, O.A., \& Argote, R.B. (2012). Motivations and barriers to compliance of obese schoolchildren with a strength training physical exercise program. Results of a qualitative study. Revista Española de Nutrición Comunitaria, 18(4), 205-210.

Wilkenfeld, R.L., Pagnini, D.L., Booth, S.L., \& King, L.A. (2008). Perceptions of adolescents on overweight and obesity: The weight of opinion study. Journal of Pediatrics and Child Health, 44(5), 248-252. doi: 10.1111/j.1440-1754.2007.01267.x

Wilmot, E.G., Edwardson, C.L., Achana, F.A., Davies, M.J., Gorely, T., Gray, L.J ... \& Biddle, S.J.H. (2012). Sedentary time in adults and the association with diabetes, cardiovascular disease and death: systematic review and meta-analysis. Diabetologia, 5, 2895-2905.

Wilson, P.M., \& Rodgers, W.M. (2002). The relationship between exercise motives and physical self-esteem in female exercise participants: An application of self-determination theory. Journal of applied biobehavioral research, 7(1), 30-43. doi: 10.1111/j.17519861.2002.tb.00074.x 